\title{
Demo Abstract: Visible Light Localization Using Incumbent Light Fixtures
}

\author{
Chi Zhang \\ University of Wisconsin-Madison \\ czhang296@wisc.edu
}

\author{
Shipei Zhou \\ Peking University \\ specialzsp@pku.edu.cn
}

\author{
Xinyu Zhang \\ University of Wisconsin-Madison \\ xyzhang@ece.wisc.edu
}

\begin{abstract}
Visible light indoor localization offers promising accuracy and reliability due to dense light deployment and multipath-free propagation. However, current visible light localization schemes rely on either energy-hungry cameras or assumptions about channel responses of light sources, which limits their application. They also require LED lights with specialized circuitry, which increases cost and hinders large scale deployment. We present LiTell2, a scheme that extracts unique features from incumbent fluorescent or LED lights, and uses a simple photodiode sensor to extract AoA information for localization. LiTell2's sensor achieves AoA measurements by leveraging the diversity of photodiodes' angular responses. By comparing signal from photodiodes of different angular response, LiTell2 can derive AoA of each light source, thus achieving high precision localization with unmodified light fixtures and extremely low fingerprinting cost. Our experiments show that LiTell2 provides accurate AoA sensing and enables energy efficient yet robust localization.
\end{abstract}

\section{INTRODUCTION}

Indoor localization is an important component in many mobile systems, enabling context-aware and location-based services and generating significant revenues in industry [1]. Accuracy, reliability and cost are among the core metrics for indoor localization systems. However, a recent evaluation of state-of-the-art localization schemes [2] shows that reliability and deployment cost problems remain as huge barriers for realistic indoor location-based services.

Emerging visible light localization schemes offer promising advantages over radio-based techniques, thanks to dense light deployment and deterministic propagation. Previous works include photodiode (PD) based [3,4] and camera based [5-7] systems, both achieving robust sub-meter level accuracy. Unfortunately, drawbacks exist in both type of systems. PD-based systems rely on RSS propagation modeling and require precise channel response of each light, which is only feasible for round lights and falls apart in practical environments with rectangular or even irregular light fixtures (where axial and lateral angular responses are different). Unlike PDs, cameras can provide AoA information and thus allow AoA-based approaches, which are more accurate [8]. However, camera-based

Permission to make digital or hard copies of part or all of this work for personal or classroom use is granted without fee provided that copies are not made or distributed for profit or commercial advantage and that copies bear this notice and the full citation on the first page. Copyrights for third-party components of this work must be honored. For all other uses, contact the owner/author(s).

SenSys '16 November 14-16, 2016, Stanford, CA, USA

(C) 2016 Copyright held by the owner/author(s).

ACM ISBN 978-1-4503-4263-6/16/11.

DOI: http://dx.doi.org/10.1145/2994551.2996534

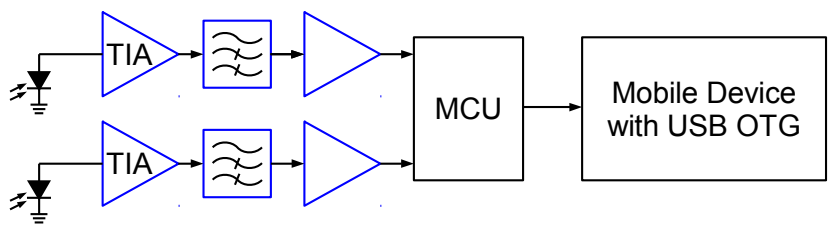

Figure 1: Diagram of the customized AoA sensor.

systems usually suffer from high energy consumption [9], high computational load, and even security concerns [10]. These drawbacks greatly limit the application of visible light localization in practical environments. More critically, existing visible light positioning systems require customized LED circuitry to generate identifiable beacons for each light bulb, leading to huge deployment cost.

To enable low-cost AoA-based visible light localization on PDbased system, we introduce LiTell2, a scheme that extracts natural fingerprints from incumbent fluorescent/LED lights, and leverages a pair of PDs to sense AoA information and allows continuous, real-time localization with PDs in practical environments. LiTell2 uses a customized light sensor (Fig. 1) to extract AoA information from visible light channel, which works by comparing information obtained from a pair of PDs with different field-of-view (FoV). The FoV difference leads to difference in RSS, which can be mapped to an AoA value. Together with orientation information from the motion sensors, equations can be formed to solve the 3 unknowns in the 3D position of the phone. LiTell2 is built on top of LiTell [7] and thus can also work with commercial unmodified fluorescent lights. However, the high SNR and dynamic range that PD provides adds support for LEDs as well (Fig. 2). We have implemented LiTell2 as an Android app for commercial out-of-the-shelf (COTS) smartphones, which has been tested on many different Android phone models. The prototype LiTell2 system shows promising performance in our experiments.

\section{OVERVIEW}

Fig. 3 shows LiTell2's overall workflow. Like LiTell, LiTell2 use commercial light fixtures as location landmarks. LiTell2's AoA sensor samples light intensity at $300 \mathrm{Ksps}$, filters out DC component that wastes dynamic range, and provides spectrum resolution down to a few Hz. This allows LiTell2 to distinguish multiple light sources, both near and far, from received light signal, since their characteristic frequencies (CF, as shown in Fig. 2) usually differ [7]. RSS of the $\mathrm{CF}$ from both PDs are then associated with each light. To figure out AoA of the light, LiTell2 compares the RSS from 2 PDs of different FoV. Since receiver's angular response is a multiplicative factor in the channel model $[3,11]$, by dividing RSS from the 2 co-located PDs, all other factors cancel out, leaving the ratio of the PDs' angular responses (Fig. 4), which can be measured during one-time factory calibration [11]. The ratio can then be mapped to an AoA value via 


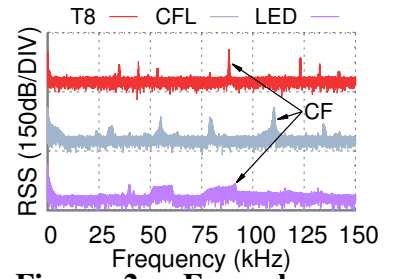

Figure 2: Example spectrum from the AoA sensor.

the calibration data. There is an ambiguous region which is unusable, but the usable FoV covers a total of approximately $50^{\circ}\left(-25^{\circ}\right.$ to $25^{\circ}$ ), similar to cameras.

To derive location from AoA information, LiTell2 uses a set of equations based on simple vector math. Since there are 3 unknowns in the device's 3D location, 3 measurements are needed, usually from 3 different lights since light deployment is dense enough in most cases. In case only one light is visible to the sensor, 3 equations can also be formed with different orientations of the phone introduced by rotation and displacement.

Like LiTell, LiTell2 also greatly simplifies the fingerprinting process since it only needs the $\mathrm{CF}$ of each light. Further, the fingerprinting can be done with the AoA sensor dongle, and with the help of Tango's motion tracking [12], location of each light can be automatically labeled. The Tango tablet is only needed during fingerprinting, and can be substituted by other devices with motion tracking ability. As a result, the fingerprinting process is unobtrusive and extremely fast. We have also prototyped a fingerprinting app (Fig. 5) that allows the user to manage the process visually.

\section{DEMONSTRATION}

In this demonstration, we will show case the prototype LiTell2 app and the AoA sensor. We will demonstrate the AoA measurement as well as localization. We will also demonstrate the fingerprinting process and its simplicity. In addition, we will also demonstrate the original LiTell [7] which uses cameras to capture characteristic frequencies of fluorescent lights.

The prototype LiTell2 app can run on any recent Android phone and requires no root privilege. The AoA sensor dongle can also be connected to recent Android phones with OTG capabilities via OTG adapters. We will provide a smartphone for the demo, but we also encourage the participants to install and try the app out on their own phones. We will also provide the Tango tablet for fingerprinting. The LiTell2 does not require Internet connection or a server since all computation is simple and self-contained.

\section{CONCLUSION}

In visible localization, camera-based triangulation systems and PD-based trilateration systems each has its own advantages and disadvantages. We present LiTell2, a system that combines the advantages of the two by augmenting AoA information to PDs, with the help of a specially designed light sensor. Experiments shows

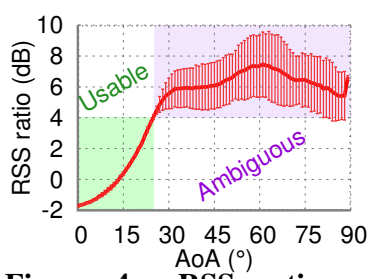

Figure 4: $\quad \operatorname{RSS}^{\circ}$

AoA.

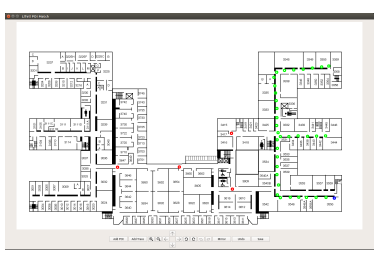

Figure 5: The fingerprinting app. that LiTell2 has promising characteristics and performance. We expect this demonstration can inspire more works based on the AoA sensing and localization technique.

\section{REFERENCES}

[1] ABI Research, "Indoor Location in Retail: Where Is the Money?" 2013.

[2] D. Lymberopoulos, J. Liu, X. Yang, R. R. Choudhury, V. Handziski, and S. Sen, "A Realistic Evaluation and Comparison of Indoor Location Technologies: Experiences and Lessons Learned," in Proc. of ACM/IEEE IPSN, 2015.

[3] L. Li, P. Hu, C. Peng, G. Shen, and F. Zhao, "Epsilon: A Visible Light Based Positioning System," in Proc. of USENIX NSDI, 2014.

[4] J. Zhang, C. Zhang, X. Zhang, and S. Banerjee, "Towards a Visible Light Network Architecture for Continuous Communication and Localization," in 3rd ACM Workshop on Visible Light Communication Systems (VLCS), 2016.

[5] Y.-S. Kuo, P. Pannuto, K.-J. Hsiao, and P. Dutta, "Luxapose: Indoor Positioning with Mobile Phones and Visible Light," in Proc. of ACM MobiCom, 2014.

[6] Z. Yang, Z. Wang, J. Zhang, C. Huang, and Q. Zhang, "Wearables Can Afford: Light-weight Indoor Positioning with Visible Light," in Proc. of ACM MobiSys, 2015.

[7] C. Zhang and X. Zhang, "LiTell: Robust Indoor Localization Using Unmodified Light Fixtures," in Proc. of ACM MobiCom, 2016.

[8] J. Armstrong, Y. Sekercioglu, and A. Neild, "Visible Light Positioning: a Roadmap for International Standardization," IEEE Communications Magazine, vol. 51, no. 12, 2013.

[9] X. Chen, Y. Chen, Z. Ma, and F. C. A. Fernandes, "How is Energy Consumed in Smartphone Display Applications?" in Proc. of ACM HotMobile, 2013.

[10] A. P. Felt, S. Egelman, and D. Wagner, "I've Got 99 Problems, but Vibration ain't One: A Survey of Smartphone Users' Concerns," in Proc. of ACM SPSM, 2012.

[11] C. Zhang, J. Tabor, J. Zhang, and X. Zhang, "Extending Mobile Interaction Through Near-Field Visible Light Sensing," in Proc. of ACM MobiCom, 2015.

[12] Google Inc., “Tango," https://get.google.com/tango/. 